\title{
Pragmatisch-integrative Geographie für Sekundarschul- Lehrpersonen? Ein konkretes Beispiel aus der Hochschullehre
}

\author{
“itta.baver@geo.uzh.ch; holger.frey@geo.uzh.ch; sara.landolt@geo.uzh.ch; andreas.linsbauer@geo.uzh.ch, \\ alle: Geographisches Institut, Universität Zürich
}

eingereicht am: 26.08.2019, akzeptiert am: 18.12.2019

Der Beitrag führt in konzeptionelle Gedanken einer „pragmatisch-integrativen Geographie“ ein und erläutert wie diese Ideen in konkreten Lehrveranstaltungen für angehende Lehrpersonen an Schweizer Sekundarschulen (Sek I), von einem Team aus Humangeographinnen und Physiogeographen in die Praxis umgesetzt werden.

Keywords: Hochschullehre, Lehrer/innenausbildung, integrative Geographie

\section{Pragmatic-integrative geography for secondary school teachers? An example from university classes}

The article introduces conceptual ideas of "pragmatic-integrative geography" and explains how these ideas are applied to teaching modules for students aiming to become secondary school teachers, in Switzerland. The lectures, as well as the text, show the fruitful cooperation of a team consisting of four geographers with research interests in human and physical geography.

Keywords: Teaching at university level, teacher education, integrative geography

\section{Einleitung}

Der „Integrations-Diskurs“ zur „Einheit der Geographie“ blickt auf eine lange Tradition in der Geographie und Geographiedidaktik zurück. Das hat einerseits fachpolitische Gründe. Andererseits kommt geographischen Instituten an Hochschulen die Aufgabe zu, angehende Lehrer/innen für das Schulfach Geographie auszubilden, die später als Geographielehrpersonen an verschiedenen Schulen das Fach in all seiner Vielseitigkeit verkörpern werden. Hierzu bedarf es einer ebenso breiten wie intensiven Fachausbildung, die von Seiten der Schule um eine praktische Ausbildungsphase ergänzt und abgerundet wird.

Anstatt die Diskursentwicklung und die verschiedenen Facetten der Debatte hier erneut zu reproduzieren (hierzu gibt es ausführliche Darstellungen u.a. von Kanwischer 2006; Müller-Mahn \& Wardenga 2005; Wardenga 2005; Wardenga \& Weichhart 2006), möchten wir mit unserem Beitrag in eine Richtung vorstoßen, die unserer Ansicht nach noch zu wenig berücksichtigt wird in der fachwissenschaftlichen wie auch geographiedidaktischen Diskussion: der gemeinsame Handlungs- und Gestaltungsraum der Hochschullehre für angehende Lehrpersonen über die „innerdisziplinären“ Grenzen hinweg. Anhand eines konkreten Beispiels aus der Modulplanung und Lehrgestaltung für angehende Sekundarschullehrpersonen des Unterrichtsfaches „Räume, Zeiten, Gesellschaften "1 (dessen Curriculum sich die Fächer Geographie und Geschichte teilen) möchten wir aufzeigen, wie eine Zusammenarbeit von Vertreterinnen und Vertretern der physischen Geographie und der Humangeographie in der Lehre konkret aussehen kann. Wir möchten einen Einblick geben, auf welcher Basis unsere Lehre am Geographischen Institut der Universität Zürich für diese Studierendenpopulation - die neben dem „Doppelfach“ „Räume, Zeiten, Gesellschaften“ in mindestens drei weiteren Unterrichtsfächern ausgebildet werden - beruht, welche Diskussionen sich ergeben und welche Vorteile und Herausforderungen sich aus so einer kooperativen Arbeit zwischen Vertreterinnen und Vertretern der physischen Geographie und der Humangeographie in der Lehre ableiten lassen.

Sekundarstufe I, 7-9. Schuljahr 


\section{Infobox: Kurzer Einblick in den Fachdiskurs zu „integrativer Geographie“}

Der z.T. sehr kontrovers diskutierte Einheitsdiskurs zur Geographie wurde von Schlüsselwörtern und Kernargumenten bestimmt, wie z. B. das „Brücken- und Integrationsfach mit ganzheitlicher Sichtweise und hoher Problemlösungskompetenz" (Wardenga \& Weichhart 2012: 1086), „die Schnittstellenwissenschaft Mensch / Natur“ (ebd.), der „Doppelcharakter der Geographi““ (Kanwischer 2006) oder die „dritte Säule der Geographie“ (Weichhart 2003). Unter der „dritten ,Säule“" versteht Peter Weichhart eine geographische Gesellschaft-Umwelt-Forschung, die ganz spezifische Fragestellungen generiert, „die in dieser Form weder in der Physiogeographie noch in der Humangeographie bearbeitet werden " und daher einer integrierenden Perspektive mit natur- und sozialwissenschaftlichen Anteilen bedürfen (Weichhart 2003: 25; Herv. i. O.).

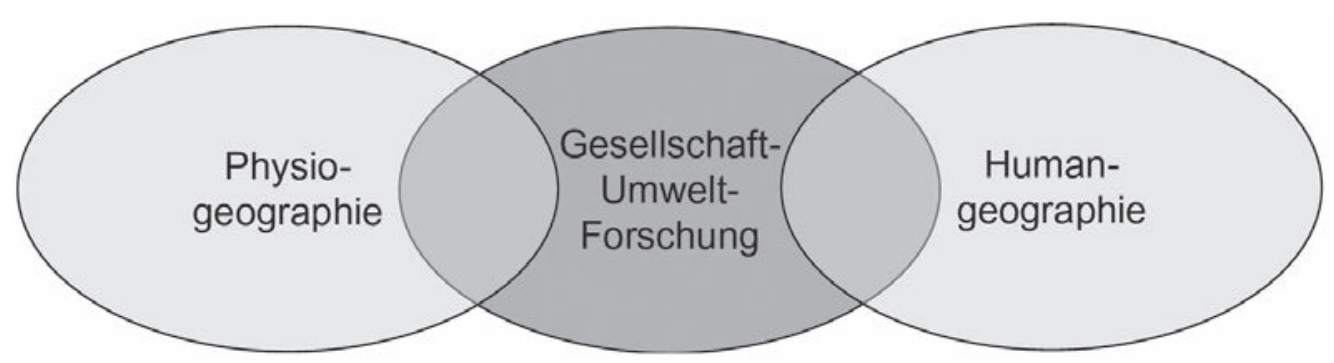

Abb. 1: Das „Drei Säulen-Modell“ der Geographie (Quelle: Weichhart 2003: 25) eigene Darstellung

Was uns aus heutiger Sicht im Zusammenhang mit der Argumentation für das „Drei Säulen-Modell“ von Weichhart zu wenig berücksichtigt erscheint, sind die in der Zwischenzeit weiter ausgebauten Bereiche der Geographie, wie z. B. die Geographische Informationswissenschaft, die Fernerkundung, virtuelle Realität oder die Integration sozialer Medien - also Techniken, Methoden und Forschungsbereiche, die sich auf die verschiedensten Forschungsbereiche innerhalb der Geographie beziehen bzw. weit über diese hinausgehen und dadurch ständig neue Kooperationen und Denkräume eröffnen.

In der deutschsprachigen Geographie wurde seit etwa der Jahrtausendwende eine vor allen Dingen „fachpolitisch motivierte Diskussion“ geführt, „in deren Rahmen eine Art New Deal zwischen den beiden Geographien gefordert wird“ (Wardenga \& Weichhart 2012: 1086; Herv. i. O.). Im Zuge ihrer Bemühungen um einen offeneren Dialog zwischen Humangeographie und physischer Geographie, der auf mehreren Tagungen und Workshops zwischen 2005 und 2007 gepflegt wurde, stellten Ute Wardenga und Peter Weichhart fest, dass „konkrete Zusammenarbeit und gemeinsame Forschung am ,Problem ' eher die Ausnahme war und ist" (2006: 12). Herauszustellen ist bei den verschiedenen Gesprächsforen in München (2003), Bonn (2004; 2007), Wien (2005), Trier (2005), Leipzig (2006), Bayreuth (2007) und den daraus entstandenen Publikationen (u. a. Heinritz 2003; Wardenga \& Weichhart 2006; Weichhart 2005) die inhaltliche Ausrichtung auf das gemeinsame Forschungspotenzial. Gemeinsame Lehrprojekte oder andere Kooperationsformen wurden in diesen Kreisen vielleicht informell verhandelt, in den schriftlichen Aufzeichnungen finden sich davon aber keine Spuren mehr. Diese Spuren nachträglich wieder sichtbar zu machen und auch neue Formen der hochschuldidaktischen Forschung und Lehre an geographischen Instituten aufzuzeigen wäre unserer Ansicht nach auch eine spannende Aufgabe für die Geographiedidaktik.

Von geographiedidaktischer Seite wurde die Diskussion zu ,integrativer Geographie“ um konzeptionelle Ansätze erweitert, die das Grundsatzproblem aus verschiedenen theoretischen Perspektiven erörterten (Gryl, Schlottmann \& Kanwischer 2015). Die Beiträge reichen hier von phänomenologischen bis hin zu systemorientierten und kompetenzorientierten Ansätzen (Dickel \& Pettig 2015; Kersting 2015; Mehren et al. 2015; Schlottmann 2015). Die theoretische wie unterrichtspraktische Herausforderung besteht aber gerade darin, den so einfach zu vermittelnden und in der Schulgeographie weithin etablierten Dualismus „Mensch-Natur" in poststrukturalistischer Façon aufzulösen und ein zeitgemäßes Verständnis von Geographie in allgemein verständlichen Worten und z. B. mit Hilfe von aktuellen sozio-technischen Beispielen für Schüler/innen begreifbar zu machen (siehe Bauer 2015; Schneider \& Wilhelm 2015). Ganz ähnlich, jedoch primär auf die Überwindung (vermeintlicher) ontologischer Differenzen abzielend, argumentiert Christian Steiner im Sinne eines pragmatischen Mensch-Umwelt-Verständnisses, das sich für eine Überwindung des dualistischen Gegensatzpaares „Mensch - Konstruktivismus“ und „Natur - Realismus“ stark macht, um den Weg für wirklich integrative Ansätze zu ebnen (Steiner 2014). 


\section{Wo wir anknüpfen - und wohin unsere Reise geht}

Sowohl in der geographischen als auch geographiedidaktischen Wissenschaftslandschaft tauchen aktuell nur noch sporadisch Beiträge auf, die sich mit der vermeintlichen „Einheit der Geographie“ kritisch auseinandersetzen (u.a. Steinbrink \& Aufenvenne 2016; Neuer \& Schlottmann 2014; Schlottmann et al. 2018). Zur „Einheitsklammer" wird hingegen auf anderen Ebenen sehr gerne und gerade dann recht häufig zurückgegriffen, wenn es darum geht, Schüler/ innen die Vielfalt der Geographie in Lehr- und Schulbüchern zu erklären (Gebhardt 2012: 3; Egli, Hasler \& Probst 2016), Studierende für heterogen angelegte Geographie-Studiengänge zu gewinnen oder Begrüßungsworte auf Geographie-Konferenzen und Schulgeographentagen zu entrichten.

Im Editorial des Rundbriefs Geographie (274) erörterten Antje Schlottmann, Boris Braun, Achim Bräuning und Christian Kuhlicke, warum aus ihrer Sicht nicht nur in der „Praxis der Hochschullehre, sondern auch und vor allem in der Forschungstätigkeit die Geographie trotz vieler Lippenbekenntnisse zur Einheit des Faches in zwei bzw. vermutlich sogar noch mehr konzeptionell, methodologisch und erkenntnistheoretisch sehr unterschiedlich strukturierte Teile (zerfällt)“" (Schlottmann et al. 2018: 1). Aus unserer Sicht stechen zwei Argumente in ihrer Diagnose hervor: Einerseits stellen sie einen „Mangel an überzeugenden integrativen Paradigmen und Theorieansätzen“ fest und zweitens eine „zunehmende Sprachlosigkeit“ bzw. Dialogmüdigkeit ,in unserem Fach selbst" (Schlottmann et al. 2018: 1).

In der Vergangenheit gab es von verschiedener Seite ernsthafte Bemühungen, einen offenen Dialog und eine gemeinsame Grundlagenforschung zu „integrativer Geographie“ anzustoßen (siehe Infobox links). Eine Verständigung darauf, was unter ,integrativer Geographie“ verstanden wird und welche Diskussionen hieraus in die verschiedenen Teilbereiche der Geographie hineinwirken könnten, sind jedoch weiterhin offene Fragen.

Vor diesem Hintergrund initiierte der Vorstand des VGDH im Mai 2018 eine Diskussionsrunde in Frankfurt zu „integrativer Geographie“. Vertreter/ innen verschiedener deutschsprachiger geographischer Institute tauschten Argumente in Bezug auf die Realisierung integrativer Geographie in Forschungsprojekten und Lehrveranstaltungen aus. Im Beitrag von Schlottmann et al. (2018) sind viele Argumente und Ergebnisse dieses Austausches zusammengefasst, wobei wir vier Aspekte hier besonders hervorheben möchten: Erstens stehen einer intensivierten $\mathrm{Zu}-$ sammenarbeit zwischen physischer Geographie und
Humangeographie sehr verschiedene Terminologien, Begriffsbedeutungen, Forschungslogiken und Erkenntnisinteressen entgegen. Zweitens lässt sich „die Zusammenarbeit über die Grenzen der Teildisziplinen hinweg theoretisch wie praktisch ohnehin nur über konkrete Themen und vor allem über gemeinsame Untersuchungsräume herstellen“. Drittens bedarf die Überwindung dieser unbefriedigenden Situation einiger Anstrengungen, wobei neben strukturellen auch „motivationale Voraussetzungen bedeutsam für eine integrative Praxis" sind. Und viertens sollte ein Austausch auf der Basis gegenseitiger „Anerkennung und Wertschätzung für unterschiedliche Denkweisen, Paradigmen, Ontologien und Forschungen über die Grenzen der Teildisziplinen hinweg" geführt werden (vgl. Schlottmann et al. 2018: 3).

Das Grundsatzdilemma „integrative oder desintegrative Geographie?" betrifft aber nicht nur die Geographie, in deren internationalen Fachkreisen neuerdings etwa die "critical physical geography“ (z. B. Lave et al. 2014; Lave, Biermann \& Lane 2018) bzw. auch die Diskussion um das „Anthropozän“ bzw. „Anthropocene" (z. B. Crutzen 2002; Johnson \& Morehouse 2014; Geist 2018; Knitter et al. 2019) die Debatte mit neuen Impulsen angeregt hat (siehe hierzu Kapitel 2.2). Auch oder gerade die Geographiedidaktik kann sich der Grundsatzdebatte schlichtweg nicht entziehen, weil sie einen konkreten Ausbildungsauftrag für die Schule bzw. das natur- und sozialwissenschaftlich angelegte Schulfach Geographie wahrnimmt. Entsprechend fällt die geographiedidaktische Diagnose von Malte Steinbrink und Philipp Aufenvenne aus: Nämlich, dass „(d)ie einst konstruierte inhaltliche Grundidee und Legitimation der wissenschaftlichen Geographie fachwissenschaftlich kaum noch salonfähig (ist); für die Schulerdkunde ist diese aber öffentlich kaum zu umgehen." (Steinbrink \& Aufenvenne 2016: 9)

Dieser recht pauschalen Diagnose - insbesondere „der" in Analogie zu Weichhart wiederum dual verstandenen Fachwissenschaft gegenüber - müssten genauere Untersuchungen folgen, die z.B. abklären würden, welche Arten (!) des wissenschaftlichen Verständnisses, welche epistemologischen Grundlagen „der“ im Plural gedachten Geographie jeweils zugrunde liegen. Ebenso müsste differenzierter auf aktuelle Diskurse in den internationalen geographischen Fachdebatten eingegangen werden. Hierdurch könnte nämlich eindrucksvoll herausgearbeitet werden, dass in letzter Zeit Geograph/innen unterschiedlichster theoretischer, methodischer und inhaltlicher Ausrichtungen (z. B. sozialökologische Ansätze, sociomateriality, critical physical geography, socio-hydrology) wieder geeignete „integrativen Fragestellungen“ "gefunden haben, die ganz bewusst dem noch von Weichhart (2005: 122) festgestellten akademischen Nebeneinan- 
derherforschen von Physiogeograph/innen und Humangeograph/innen eine engagierte und tatsächlich „integrierende Wissenschaftspraxis“ entgegenstellen. Ein so verstandener wissenschaftlicher Freiraum, der neue Wege der Kommunikation und Kooperation auslotet, erscheint uns vielversprechend.

Einer analogen Ausdifferenzierung bedürfte es unserer Ansicht nach ebenso für "die“ Geographiedidaktik. Wir sind uns durchaus bewusst, dass die Beziehung zwischen Fachdidaktik und Fachwissenschaft keine einfache war und ist. Sie ist vorbelastet durch einen Diskurs, in dem es „viel um gegenseitige Anerkennung und Wertschätzung geht“, wobei sich „,die’ Fachdidaktik von ,der' Fachwissenschaft oft nicht ernst genommen fühlte" (Neuer \& Schlottmann 2014: 1) und entsprechend unter fehlender Wertschätzung und Anerkennung (vgl. Steinbrink \& Aufenvenne 2016: 6) litt. Das ist jedoch ein sehr negatives Selbstbild. Es liegt an Geographiedidaktikerinnen und -didaktikern dem ein positiveres Bild entgegenzusetzen und selbstbewusst die eigenen Forschungsanliegen und das Selbstverständnis einer modernen Geographiedidaktik herauszustellen. Birgit Neuer und Antje Schlottmann gehen in diese Richtung, wenn sie vorschlagen, dass sich die Geographiedidaktik als „forschende Disziplin mit spezifischen Fragestellungen begreift" und dabei ein Erkenntnisinteresse und Forschungsfeld ,jenseits des reinen Schulbezugs" fokussiert, das über die Inhalte der unterrichtsmethodischen, praxisorientierten Lehrer/innenausbildung hinausgeht (vgl. Neuer \& Schlottmann 2014: 1; siehe auch Argumente von Eva Nöthen und Verena Schreiber in Bauer \& Gryl 2018).

\section{Positionierung einer ,pragmatisch-integ- rativen Geographie“}

Gerne möchten wir an den Grundgedanken des Textes von Schlottmann et al. (2018), der aus einer Kooperation von drei Geographen und einer Geographin mit verschiedenen fachlichen Interessenrichtungen entstanden ist, anknüpfen und erläutern, was wir unter einer "pragmatisch-integrativen Geographie“ verstehen und wie wir diese Leitideen in gemeinsamen Lehrveranstaltungen umsetzen. Hierzu bedarf es aber erst einmal der Offenlegung unserer verschiedenen, fachlichen Hintergründe und Perspektiven als Geographinnen und Geographen.

\subsection{Unsere individuellen Sichtweisen}

"Meine Forschungsarbeit als Geograph fokussiert auf Hochgebirgsregionen und beschäftigt sich mit den Wechselwirkungen zwischen verschiedenen Prozessbereichen und dem Klima. Als Naturwissen- schaftler gehe ich dabei von den naturräumlichen Prozessen aus, interessiere mich aber auch dafür, wie diese Prozesse sich auf das Leben der Menschen auswirken. Gerade bei Bestrebungen zur Naturgefahrenprävention oder Maßnahmen zur Anpassung an den Klimawandel ist die Integration der betroffenen Menschen und Institutionen absolut entscheidend. Um nachhaltig wirksame Ergebnisse zu erhalten, müssen darum klassische humangeographische Themen, wie die sozioökonomischen und kulturellen Hintergründe der betroffenen Bevölkerung, mit den physisch-geographischen Aspekten der involvierten naturräumlichen Prozesse kombiniert werden. Aus meiner Sicht ist darum der integrative Ansatz eine Voraussetzung für eine moderne und lösungsorientierte Geographie." (Holger)

„Grundsätzlich stimme ich dir zu. Ich finde aber, dass ein integrativer Ansatz nur für bestimmte Themenbereiche der Geographie geeignet ist. Zusätzlich möchte ich aber einen weiteren Punkt hervorheben. Als feministische Humangeographin, die Freude an theoretischen Ansätzen hat, ist mir wichtig, dass wir nicht nur versuchen Integration über Themen herzustellen, sondern integrative Geographie auch als etwas denken, das von unseren unterschiedlichen theoretischen Zugängen profitieren kann. Dabei sollten wir uns nicht von unseren $z$. T. sehr unterschiedlichen Paradigmen und Vokabularen abschrecken lassen, sondern uns Zeit nehmen, andere Positionen anzuhören, versuchen $z u$ verstehen, warum diese eingenommen werden und in einen konstruktiven Dialog zueinander treten. "(Sara)

„Aus meiner eigenen Forschungstätigkeit, die sich intensiv um die mediale Aufbereitung und Kommunikation von Gletscherveränderungen im Zusammenhang mit dem Klimawandel, die damit einhergehenden Veränderungen im Hochgebirge, sowie deren Auswirkungen auf die Menschen befasst, kann ich bestätigen, dass dies tatsächlich möglich ist. Da Gletscher sehr empfindlich auf die globale Erwärmung reagieren, werden sie oft als Sinnbild für den Klimawandel ins Feld geführt. Das Interesse an Gletscherveränderungen und der Erklärungsbedarf ist daher groß. In einem laufenden Projekt geht es darum den Klimawandel in virtueller Realität anhand einer Zeitreise über 200 Jahre am Aletschgletscher $z u$ erläutern (www.expedition2grad.ch). Dieses Projekt - eine Zusammenarbeit von Wissenschaftlerl innen aus physischer und Humangeographie, Künstlerlinnen, Padägog/innen und Museumsexpert/innen - zeichnet sich durch seine Interdisziplinarität aus. Alle beteiligten Personen haben ihr Know-how aus den verschiedensten Bereichen und aus den un- 
terschiedlichsten Perspektiven eingebracht, um das Projekt zu verwirklichen. Wir haben es geschafft eine gemeinsame Sprache zu finden, aber ja, Zeit hat das gebraucht. Ich sehe mich dabei als Geograph, auch wenn ich mir nicht immer den Kopf darüber zerbreche, was die Geographie denn so ist." (Andreas)

"Ich möchte an den Gedanken von Andreas anschließen, weil sich hier eine gemeinsame Erfahrung von Geographen und Geographinnen abzeichnet unabhängig ob sie jetzt an der Universität forschen und lehren oder aber an Schulen unterrichten. Im Kern geht es in , der Geographie' doch um die Sache und nicht um die Beschäftigung mit sich selbst. Ich provoziere weiter: Sind uns gute Schulen vielleicht sogar einen Schritt voraus? Ich meine, die Basis der gemeinsamen Arbeit in einem interdisziplinär angelegten Schul-Kollegium ist doch recht klar: Es geht darum, Kinder und Jugendliche nach ihren Neigungen und Begabungen bestmöglich zu fördern und individuelles und gemeinsames Lernen aus einem Zusammenspiel von Können, Wissen und Wollen heraus zu begreifen und zu gestalten. Ob Geographie, Räume-Zeiten-Gesellschafen oder Natur und Technik auf der Stundentafel steht, das mag in politisch-strategischen Kreisen von großer Bedeutung sein. Inhaltlich viel wichtiger erscheint mir jedoch, dass es der Schule gelingt, Kinder für (geographische) Themen und Phänomene nachhaltig zu begeistern, weil sie sich dadurch neue Horizonte erschließen können. Dasselbe trifft ja auch auf unsere Lehre für angehende Sekundarschullehrkräfte zu. Und hier erscheint mir ein pragmatisch-integrativer Ansatz einerseits konzeptionell wertvoll; andererseits schlagen wir vor, die Praxisseite gleichberechtigt mitzudenken und ,pragmatisch-integrative Geographie' in LehrLernsettings konkret umzusetzen." (Itta)

\subsection{Was ist „pragmatisch-integrative Geographie“ für uns?}

In den persönlichen Positionen in Bezug auf „pragmatisch-integrative Geographie" schwingen sehr unterschiedliche Erwartungen mit, welche auch durch Erfahrungen aus (interdisziplinären) Forschungsprojekten genährt werden. Eine große Herausforderung für die Geographie im Schulunterricht zeichnet sich dadurch aus, dass eine Lehrperson für die ganze Komplexität und Breite des Faches einstehen muss. Wir sind uns dieser Situation durchaus bewusst, wenn wir Geographie-Module für angehende Sekundarschullehrpersonen unterrichten. Zumal diese Lehrerinnen und Lehrer ein breites Spektrum an Fächern unterrichten werden, in denen sie jeweils eine kompakte Ausbildung in Fachwissenschaft und Fachdidaktik absolviert haben.
Die gemeinsame Basis für unseren Dialog und unsere Lehrtätigkeit ist eine grundsätzlich offene und pragmatische Haltung gegenüber kooperativen Lehrformen und ein Interesse verschiedene geographische Positionen und Perspektiven im Team zu diskutieren und in unseren Lehrveranstaltungen bei geeigneten Themen eine „integrierende“ Sichtweise auf Geographie in der Praxis anzuwenden. Eine solche Offenheit wiederum zu erreichen, so deuten unsere dargelegten Perspektiven an, ist anspruchsvoll, benötigt sie doch viel Zeit für Diskussionen und Klärungen, gegenseitige Anerkennung und eine Sensibilität für Machtund Dominanzverhältnisse, genau dann, wenn unterschiedliche Perspektiven miteinander in Diskussion treten. Diese unterschiedlichen Perspektiven beruhen auf der Sozialisierung mit sehr verschiedenen theoretischen Debatten, methodischen Verfahren und Vorstellungen in Bezug auf geographische Forschungsinteressen. Einen Schritt weiter zu gehen und zusammen Fragestellungen jenseits von eng gezogenen Disziplingrenzen $\mathrm{zu}$ entwickeln, ist spannend, aber auch zeitaufwendig und nicht immer konfliktfrei. Zudem braucht es Gelegenheiten, Zeitgefäße und den Willen sich darauf einzulassen.

In internationalen Fachdebatten passiert aber genau das: Dialoge über Disziplingrenzen hinweg, um neue Fragestellungen und Lösungsansätze zu entwickeln. Allerdings haben sich zu potenziell integrativen Themen, wie etwa dem Klimawandel, bislang Naturwissenschaftler/innen sehr viel prominenter zu Wort gemeldet als Sozialwissenschaftler/innen. Noel Castree (2015) widmet sich diesem Problem und untersucht in einem Artikel die Beiträge und Beziehungen von Geographinnen und Geographen in der vorwiegend von Geowissenschaftlerinnen und -wissenschaftlern, und „environmental social scientists “ geprägten Debatte rund um das Anthropozän. Für Noel Castree umfasst das „Anthropo(s)cene ... the networks, institutions and publications devoted to comprehending and responding to a fast-changing Earth departing from Holocene boundary conditions." (Castree 2015: 301). Er stellt fest, dass in letzter Zeit die Geowissenschaften immer mehr versuchen auch sozialwissenschaftliche Aspekte in ihre Studien zu integrieren. Ein Trend, den Holger in seinem Forschungsnetzwerk ebenfalls bestätigen kann. Die Hauptproblematik an dieser Entwicklung sieht Castree darin, dass dadurch vor allem jene Forschungsbereiche der Sozialwissenschaften „integriert“ werden, die eine den Naturwissenschaften nahestehende analytisch-quantitative Forschungsausrichtung vertreten oder sich für eine zielgruppenspezifische Kommunikation der ,interdisziplinären" Forschungsergebnisse am besten eignen (Castree 2015: 305). Engagierten Geographinnen und Geographen, wie Eric Lambin, Diana Liverman 
und Tim Lenton, die maßgeblich an der konzeptionellen Entwicklung der "planetary boundaries idea“ (Rockström et al. 2009) und großen Forschungsverbünden (IGBP und IHDP) beteiligt waren, und einer neuen Generation an sozial-und-naturwissenschaftlich interessierten Nachwuchsforschenden (die nicht namentlich angeführt werden), traut er durchaus zu, die neuen großen Forschungsnetzwerke (wie Future Earth; https://futureearth.org) proaktiv mitzugestalten. In diesem Zusammenhang hat es möglicherweise auch Raum für kritische Alternativen zum Mainstream, die zu neuen Fragestellungen führen. Zum Beispiel: Welche Annahmen liegen der Definition von Ursache und Effekt in Bezug auf Klimawandel und menschlichem Verhalten oder Handeln zugrunde? Wie objektiv und neutral sind Klimamodelle? Welche politischen Effekte und gesellschaftliche Relevanz haben diese? (vgl. Castree 2015: 308) Zu ähnlichen Ergebnissen kommen Wesselink et al. (2017) bezüglich des Themas "Gesellschaft und Wasser", indem sie Überlegungen anstellen, wie „socio-hydrology and hydrosocial analysis" besser miteinander in Dialog treten und gemeinsam aktuelle Fragen angehen können.

Wir möchten diese Argumente aufnehmen und uns in diesem Sinne für eine machtsensible sach- und problembezogene Kollaboration unter Physio- und Humangeographinnen und -geographen einsetzen, weil es aus unserer Sicht in einer pragmatisch-integrativen Geographie genau darum geht. Was aus der angloamerikanischen Debatte für uns deutlich wird, ist die Tatsache, dass es hier nicht um eine selbst-reflexive Beschäftigung der Beziehung zwischen Humangeographie und physischer Geographie geht, sondern darum, welchen Beitrag Wissenschaftler/innen mit unterschiedlichen Forschungsinteressen zu einem Problem, einem Phänomen oder einer akademischen Debatte mit einer hohen gesellschaftlichen Relevanz beitragen können. Das ist für uns ein klares Unterscheidungsmerkmal zur deutschsprachigen Debatte innerhalb der Geographie und ein Argument für eine neu ausgerichtete Art der „pragmatisch-integrativen Geographie". Eine Perspektive, die nicht lange danach fragt, wie sie sich selbst verstehen möchte, sondern vielmehr selbstbewusst auftritt und anpackt: Was können wir damit tun? Wohin kommen wir mit dieser Perspektive? Wo fangen wir an und bis wohin kommen wir in unserem Denken? Worin liegen die wissenschaftlichen Herausforderungen der Zukunft? Wir sehen unseren Ansatz der pragmatisch-integrativen Geographie im Sinne dieses Verständnisses einer sach- bzw. problembezogenen, interdisziplinären Kollaboration, die uns in der aktuellen Lehrtätigkeit für Sekundarschul-Studierende vor die Herausforderung stellt, diesen Ansatz nicht nur in der Theorie zu entwickeln, sondern diese Ideen gleich in die Praxis umzusetzen.

\section{Das Thema „Wasser“ in unseren Grund- und Vertiefungsmodulen}

Lehrpersonen, die an Sekundarschulen in der Deutschschweiz auf Sekundarstufe I unterrichten, sind keine Fachlehrkräfte. Vielmehr decken sie einen umfassenden Fächerkatalog von vier Fächern ab, zu denen sie in der Ausbildung jeweils eine fachwissenschaftliche und fachdidaktische Grundausbildung erhalten haben. Zukünftige Sekundarschullehrpersonen, die sich an der Pädagogischen Hochschule in Zürich ausbilden lassen und als eines ihrer zukünftigen Unterrichtsfächer „Räume, Zeiten, Gesellschaften“ wählen (die Population unseres Artikels), besuchen maximal vier fachwissenschaftliche Geographie-Module mit je 3 ECTS. Dazu kommen fachdidaktische Module (Umfang Fachdidaktik Geographie und Geschichte zusammen: 22 ECTS) (vgl. PHZH o.J.). Die Studierenden, die unsere fachwissenschaftlichen Geographie-Module besuchen, haben also ein klares Berufsziel vor Augen: Sie sehen sich als Lehrpersonen für Sekundarschüler/innen und nicht als Geographielehrpersonen. Diesen angehenden Sekundarschullehrpersonen bieten wir mit unseren Modulen ein fachwissenschaftliches Kurzstudium in Geographie an, das ein Fundament zu Themen, Konzepten und Methoden der Geographie legen soll. Die beiden Module "Grundkurs Geographie 1“ und „Grundkurs Geographie 2" (je 3 ETCS) besuchen alle Studierenden, die das Unterrichtsfach „Räume, Zeiten, Gesellschaften“ gewählt haben. Die beiden fachwissenschaftlichen Vertiefungsmodule (je 3 ECTS) können wahlweise im Bereich Geographie oder Geschichte belegt werden. Die überwiegende Mehrheit unserer Veranstaltungen (Grund- und Vertiefungskurse) ist so gestaltet, dass die Studierenden pro Doppellektion (90 min.) entweder ein humangeographisches Thema oder einen physisch-geographischen Schwerpunkt behandeln. Diese Doppellektionen werden in Gruppen von 20-25 Studierenden unterrichtet; entsprechend den Studierendenzahlen werden diese Doppellektionen in den Grundkursen vier Mal, in den Vertiefungskursen zwei Mal gehalten (vgl. Abb. 2 und 4).

Anhand des Themas „Wasser“ möchten wir nun konkreter aufzeigen, welche Chancen und Herausforderungen sich für Lehrveranstaltungen ergeben, die wir nach dem Prinzip einer „pragmatisch-integrativen Geographie" aufgebaut haben. Bei der Planung und Umsetzung der gemeinsamen Hochschullehre (Gesamtcurriculum, Themensequenzen, Lernzielkontrollen) wollten wir den Studierenden ganz bewusst auch geeignete Beispiele vorstellen, die dem Prinzip einer "pragmatisch-integrativen Geographie“ folgen. Das Entwickeln des Curriculums für die Lehre erfordert ein Miteinander im Team, bei dem alle ihre Gedan- 
ken, Haltungen und Ideen einbringen. Das bedingt, dass wir wohlwollend miteinander sprechen, Standpunkte gegenseitig erläutern, Dinge aushandeln und gewisse Differenzen auch stehen lassen können. Dabei geschieht sehr viel „pragmatisch-integrative Geographie" auf der Hinterbühne, was später auf der Vorderbühne, also im Unterricht, nicht explizit als solche erkennbar ist oder verkörpert wird. So steht etwa, auch aus Ressourcengründen, meist nur eine Person im Unterrichtsraum ${ }^{2}$, die basierend auf ihrer Ausbildung, ihrem Forschungs- und Lehrschwerpunkt der Physio- oder Humangeographie zugeordnet werden kann, lehrt aber je nach Kurs und Thema im Sinne einer pragmatisch-integrativen Geographie.

Die beiden Module Grundkurs Geographie 1 \& 2 sind für die angehenden Sekundarschullehrpersonen, die das Schulfach „Räume-Zeiten-Gesellschaften“ (RZG) auf Sekundarstufe I unterrichten werden, obligatorisch und dauern zusammen zwei Semester. Entlang von verbindlichen Lernzielen werden aus den Themenbereichen Physische Geographie und $\mathrm{Hu}-$ mangeographie verschiedene fachwissenschaftliche Grundlagen und Zugänge vorgestellt und eingeübt. Es kommen grundlegende Methoden und Techniken des wissenschaftlichen Arbeitens an relevanten Inhalten und Forschungsthemen der Geographie zur Sprache. Diese Kurse sind den fachwissenschaftlichen Grundlagen gewidmet, nicht der Fachdidaktik (siehe Abb. 2). Die Themen der Grundkurse werden in den meisten Fällen „klassisch“ getrennt nach Human- und Physiogeographie unterrichtet. Neben einigen Lektionen, die der methodischen Geographie zuzuordnen sind, werden gewisse Themen, wie zum Beispiel der Klimawandel, sowohl aus human- als auch aus physisch-geographischer Perspektive behandelt, wobei auch aktiv Querbezüge hergestellt werden.

Das Thema "Wasser" dient uns im Folgenden als Beispiel, um zu zeigen, wie ein Thema aus physischund humangeographischer Perspektive mit den entsprechenden Querbezügen im Grundkurs $1 \& 2$ be-

2 Bedenkt man, dass die Grundkurse vierfach durchgeführt werden, spitzt sich die Frage der personellen Ressourcen bei der Anwesenheit mehrerer Dozierender in einer Lektion nochmals zu. handelt und aus pragmatisch-integrativer Perspektive im Vertiefungskurs 1 wieder aufgenommen wird.

\subsection{Wasser im Grundkurs $1 \& 2$ - Physische Geographie}

Im ersten Semester des Grundkurses nimmt Wasser in den Veranstaltungen zur Klimatologie (Planetarische Zirkulation) und Meteorologie (Typische Wetterlagen im Alpenraum) bereits eine zentrale Rolle ein. Im zweiten Semester folgen Doppellektionen zu „Wasserkreislauf und Ozeane“ sowie "Wasser in der Schweiz“. Dabei werden die Studierenden von einem globalen Maßstab der Wasserthematik zu einer lokaleren Betrachtung hingeführt. In der ersten Doppellektion geht es vor allem darum, die physischen Faktoren der Wasserverfügbarkeit, den Wasserkreislauf als solches sowie die Faktoren der Meeresströmungen und Meereszirkulation zu erfassen. Das spiegelt sich auch in den Lernzielen dieser Doppellektion wider.

Ausgewählte Lernziele:

- Sie kennen die globale Verteilung der Wasserressourcen.

- Sie können die am Wasserkreislauf beteiligten Komponenten nennen und erklären.

- Sie sind in der Lage für ein Einzugsgebiet eine Wasserhaushaltsgleichung, welche alle Komponenten berücksichtig, aufzustellen.

- Sie wissen über den Wärmetransport der Meeresströmungen und deren Auswirkungen auf die Landmassen Bescheid (Golfstrom, Küstenwüsten).

- Sie können die thermohaline Zirkulation und das ozeanische Förderband beschreiben.

Die Studierenden erfahren, wie „klein“ die Sphäre des irdischen Wassers ist, wie „wenig“ Süßwasser im globalen Kreislauf als nutzbares Wasser den Menschen zur Verfügung steht, dass diese Ressourcen durch physische Faktoren global unterschiedlich verteilt sind und, dass nicht nur physische Faktoren die Ursache von Wasserknappheit sein können. Der globale Wasserkreislauf wird anhand der verschiedenen Komponenten durchgespielt und durchgerechnet und der

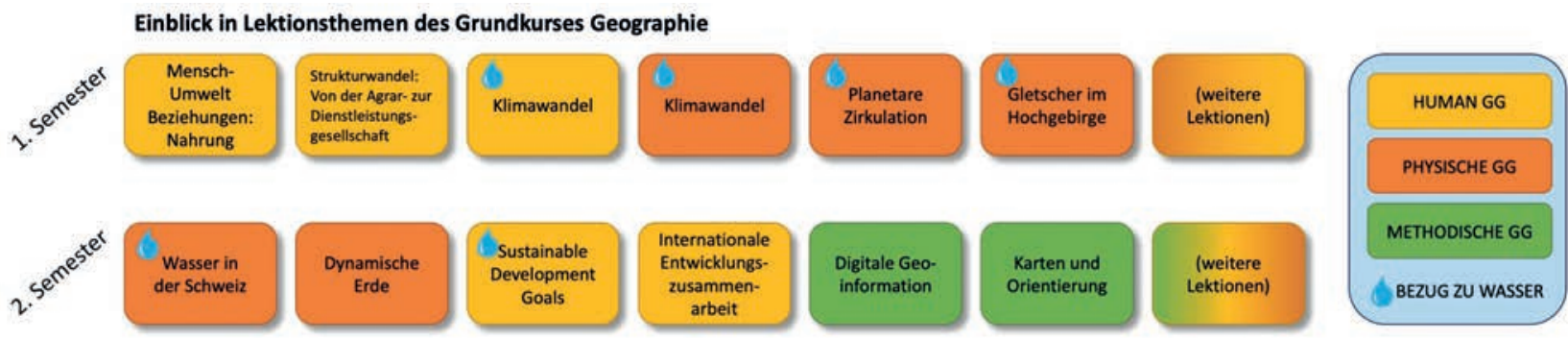

Abb. 2: Eine Auswabl der in den Grundkursen 1 und 2 behandelten Themen, wobei diejenigen mit Bezug zum Thema "Wasser" als solche gekennzeichnet sind. Ein Thema entspricht einer Doppellektion. 
sich daraus ergebende Unterschied zwischen ariden und humiden Gebieten wird erarbeitet. Anhand von Niederschlag, Verdunstung, Abfluss und Speicherung wird in einem kleineren Einzugsgebiet das Konzept der Wasserbilanz vorgestellt und geübt.

Im zweiten Teil geht es um die ozeanischen Strömungen, deren Antriebskraft und Transport von Wärme und Energie. Der Golfstrom wird etwas detaillierter besprochen und dient als Einstieg in die thermohaline Zirkulation, welche das globale Förderband der Meeresströmungen antreibt.

Das zentrale Element ist „der Kreislauf“, sei es der globale Wasserkreislauf, die thermohaline Zirkulation oder eine Wasserbilanzgleichung. Die Studierenden sollen erkennen, dass die Menge an Wasser gegeben ist, sich aber in einem Kreislauf befindet. Anhand von Flussdiagrammen und (Welt-)Karten werden diese Zusammenhänge erarbeitet und geübt.

In der darauffolgenden Doppellektion wird die physisch-geographische Komponente der Thematik Wasser am Beispiel der Schweiz auf einem lokalen Maßstab veranschaulicht und vertieft.

Ausgewählte Lernziele:

- Sie kennen die räumlichen und zeitlichen Komponenten von Niederschlag und Abfluss in der Schweiz.

- Sie kennen die drei Haupttypen der Schweizer Abflussregime und können diese erklären.

\section{Niederschlag (Herkunft des Wassers)}

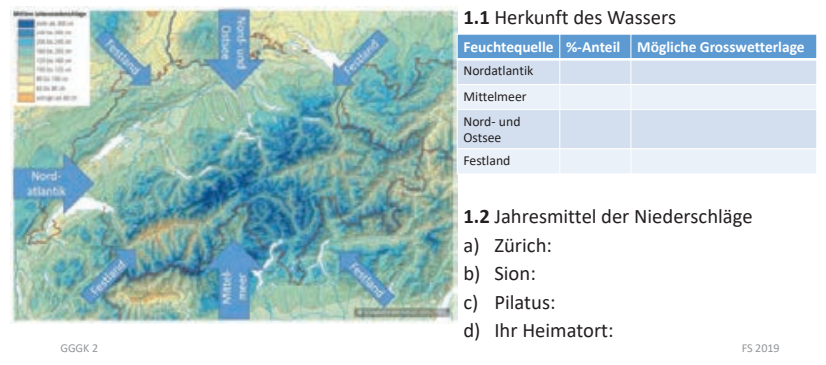

\section{Wasserbilanz der Schweiz}

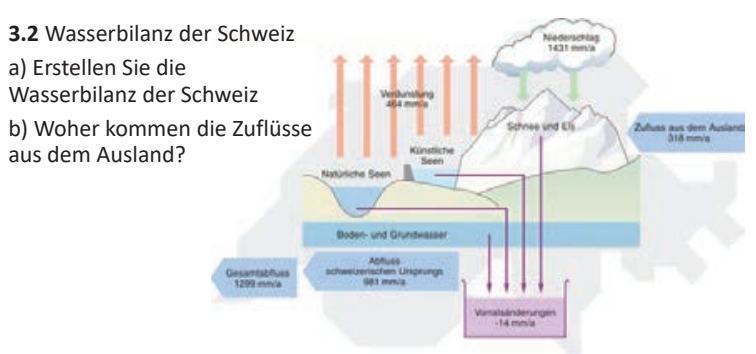

- Sie können einen Hydrographen (Pardé-Koeffizienten) lesen und interpretieren.

- Sie können den Einfluss des Klimawandels auf den Wasserhaushalt eines alpinen Einzugsgebietes beschreiben.

Als Einstieg werden zwei Kernfragen bearbeitet: Woher und wie kommt das Wasser in die Schweiz? Wie und wann verlässt das Wasser die Schweiz? Der Fokus der Doppellektion liegt auf der räumlichen und zeitlichen Komponente von Niederschlag und Abfluss. Die Studierenden analysieren raum-zeitliche Muster, die sich daraus ergeben. Das führt in einem nächsten Schritt zur Wasserbilanz der Schweiz, in welcher die Rolle der Gletscher als Speicher hervorgehoben wird. Bedingt durch das Relief und die lokalen klimatischen Bedingungen (Temperatur und Niederschlag), können die Abflussregimes der Schweiz den drei Typen pluvial, nival und glazial zugeordnet werden. Abschließend wird erarbeitet, wie sich die Abflussregime in Folge des Klimawandels (z. B. Gletscherrückgang) verändern werden.

Graphische Darstellungen auf Karten oder in Diagrammen dienen dazu, die zentralen Elemente des Wassers in der Schweiz zu erlernen. Das Interpretieren einer räumlichen Abflusskarte der Schweiz, das Zustandekommen eines Hydrographen und die Interpretation desselben sind dabei wichtige Elemente (siehe Abb. 3).

\section{Abfluss (der Schweiz)}

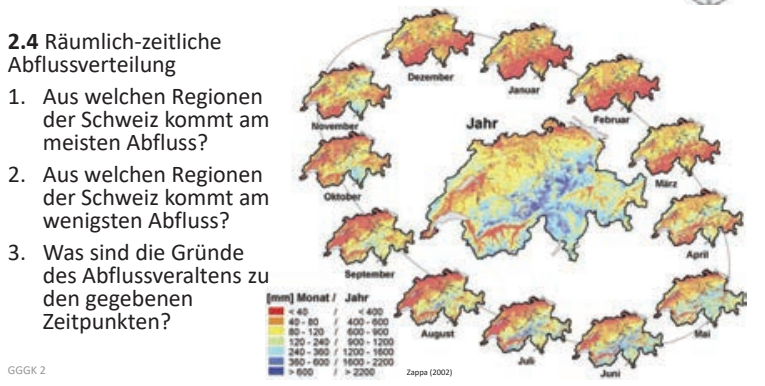

\section{Abflussregime der Schweiz}

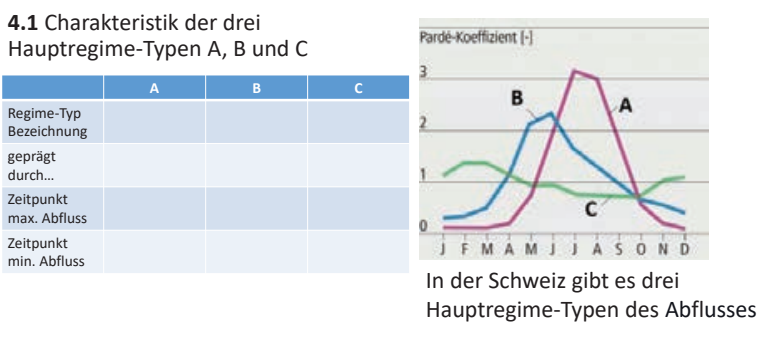




\subsection{Wasser im Grundkurs 1 \& 2 - Humangeographie}

In den humangeographischen Veranstaltungen der Grundkurse wird "Wasser" im Kontext der "Sustainable Development Goals" (SDGs) in einer Doppellektion thematisiert. Das Ziel besteht darin, dass die Studierenden die Idee hinter den SDGs kennenlernen und sich vertieft mit der Thematik des SDG 6 ,sauberes Wasser und Sanitäreinrichtungen“ auseinandersetzen. Wie alle SDGs ist auch das SDG 6 sehr umfassend. Es beinhaltet Ziele im Bereich des gerechten Zugangs zu einwandfreiem und bezahlbarem Trinkwasser, wie auch Ziele im Bereich des Zugangs zu einer angemessenen und gerechten Sanitärversorgung und Hygiene oder im Bereich der Effizienzsteigerung der Wassernutzung in allen Sektoren (UN o. J.). Ein Teil dieser thematischen Vielfalt spiegelt sich auch in den Lernzielen der Doppellektion wider.

\section{Ausgewählte Lernziele:}

- Sie kennen den Begriff, „Sustainable Development Goals" (SDG, Nachhaltige Entwicklungsziele bis 2030) und können diesen in der entwicklungspolitischen Debatte verorten.

- Das SDG 6 fordert: „Gewährleistung des Zugangs zu Wasser und sanitären Anlagen und Abwassersystemen für alle." Sie können mit ausgewähltem Fach- und Faktenwissen aus sozialer, ökologischer und ökonomischer Perspektive argumentieren, warum dieses Ziel hohe Relevanz hat.

- Sie kennen Interventionen zur Verbesserung der Sanitärversorgung und der Siedlungshygiene.

- Es wird davon ausgegangen, dass der globale Wasserverbrauch in den nächsten Jahren zunimmt. Sie können erklären, welches die Gründe hierfür sind.

In der Doppellektion bekommen die Studierenden eine kurze Einführung $\mathrm{zu}$ den SDGs, wobei der Fokus auf das SDG 6 ,sauberes Wasser und Sanitäreinrichtungen" gelegt wird. Anschließend erfolgen - ausgehend von Leitfragen - Diskussionen in Kleingruppen von im Vorfeld gelesener Texte. Die Diskussionen in der Kleingruppe sind in Anlehnung an die Gruppenpuzzlemethode aufgebaut. Das heißt, der eine Teil der Studierenden hat das Textpaket A, der andere Teil das Textpaket B im Vorfeld gelesen. In der Lektion selbst erfolgt der Austausch zuerst in homogenen Kleingruppen (inkl. der Beantwortung von Fragen zum jeweiligen Textpaket). Anschließend tauschen sich jeweils eine Person mit Textpaket A und eine mit Textpaket $B$ entlang der Leitfragen aus. Dabei setzen sich die Studierenden einerseits mit virtuellem Wasser und dem Wasserverbrauch auf globaler Ebene, anderseits - anhand konkreter Fallbeispiele aus dem globalen Süden - mit dem Zugang zu Wasser, Auswirkungen fehlender Sanitärversorgung (z.B. fehlende Toiletten) und Investitionen in die Wasserund Sanitärversorgung auseinander.

Bei den Texten handelt es sich einerseits um gut verständlich geschriebene fachwissenschaftliche Texte, anderseits um kurze Internet-Texte der Schweizer NGO Helvetas, einer unabhängigen Organisation für Entwicklungszusammenarbeit, die in Afrika, Asien, Lateinamerika und Osteuropa Hilfe zur Selbsthilfe leistet (Helvetas 2019). Bei der Auswahl der Texte und den Fragen zu den Texten wurden mehrere Ziele verfolgt:

Erstens sollte einer der Texte die Breite des Themas Wasser aufzeigen. Idealerweise sollten dabei auch Aspekte angesprochen werden, welche die Studierenden in den vorhergehenden Doppellektionen bearbeiteten, in denen Wasser aus einer physisch-geographischen Perspektive behandelt wurde. Ziel ist es, auf diese Weise die aktuelle humangeographisch ausgerichtete Doppellektion zu Wasser mit den vorherigen physischen Lektionen zu verbinden und zugleich das bisher Erlernte zu aktivieren. Konkret erfolgte eine Aktivierung des bereits Gelernten, indem für die Beantwortung der Fragen zum Text Wissen aus der letzten Lektion relevant war.

Zweitens sollten die Texte Themen behandeln, die im SDG 6 explizit angesprochen werden. Dies wird beispielsweise dadurch eingelöst, dass die Thematik des Zugangs zu Toiletten behandelt wird. Ein Thema das im SDG 6.2 explizit angesprochen wird. ${ }^{3}$ In zwei Texten wird diesbezüglich auch aufgezeigt, dass ein fehlender Zugang zu Toiletten für unterschiedliche Personen (z. B. ältere Personen, männliche/weibliche Personen, kranke Personen, von Armut betroffene Personen, Kinder) unterschiedliche Implikationen haben kann. Ein aus unserer Sicht sehr bedeutender Punkt, da dadurch die Thematik der Ungleichheit und Ressourcenausstattung als auch -zugang aufgenommen wird, die weit über das Lektionsthema SDG 6 „sauberes Wasser und Sanitäreinrichtungen" hinaus von großer Relevanz für weitere geographische Fragestellungen ist.

Drittens war es uns wichtig, dass die Texte unterschiedliche Stimmen und Perspektiven repräsentieren. Konkret sollten a) wissenschaftliche Texte wie auch aktivistische und institutionelle Texte zum Zuge kommen, b) mindestens eine Autorin unter den Autorinnen und Autoren sein und c) es sollten die Stimmen betroffener Personen zumindest in einem der Texte vorkommen, denn in feministischen und postkolonialen Beiträgen wird zu Recht darauf verwiesen, dass

„6.2: Bis 2030 den Zugang zu einer angemessenen und gerechten Sanitärversorgung und Hygiene für alle erreichen und der Notdurftverrichtung im Freien ein Ende setzen, unter besonderer Beachtung der Bedürfnisse von Frauen und Mädchen und von Menschen in prekären Situationen“ (EDA 2017). 
die tertiäre Lehre nach wie vor von Sichtweisen weißer Männer dominiert wird.

\subsection{Zwischenfazit Wasser im Grundkurs 1 \& 2}

Innerhalb der beiden Grundkurse haben die Studierenden sich mehrmals mit der Thematik "Wasser“ auseinandergesetzt. Dabei fällt auf, dass dies meist aus einer physisch-geographischen Perspektive geschah. Da wir als Dozierende für die Studierenden recht deutlich jeweils „die“ Humangeographie, respektive „die“ Physische Geographie verkörpern, müssen wir wohl davon ausgehen, dass wir "Wasser“ durch unseren Unterricht im Grundkurs eher zum Thema „der“ Physischen Geographie gemacht haben. Dass Aspekte des Themas "Wasser" von den Studierenden jeweils klar einer der beiden Richtungen zugeteilt wird, zeigte sich beispielsweise in der folgenden Interaktion mit einem Studierenden. In der Doppellektion zum Klimawandel (Fokus Klimawandel und Gesellschaft) mit einer Person von uns, die humangeographische Themen unterrichtet, tauchten bei einem Studenten Fragen auf, wie genau das Abschmelzen des Eisschildes in Grönland zum Meeresspiegelanstieg beitrage bzw. wo der Meeresspiegel um wie viel Zentimeter ansteige, ob es da regionale Unterschiede gäbe, und wenn ja, welche und wie diese erklärt werden würden. Noch bevor die Humangeographin leer schlucken konnte, schloss der Student seine Frage mit folgenden Worten ab: „Ich glaube, ich frage das nächste Woche, da haben wir physische Geographie.“

\subsection{Wasser im Vertiefungskurs}

Im Vertiefungskurs Geographie 1, mit dem Titel „Welt im Wandel“, behandeln wir, aufbauend auf den Grundlagen der Grundkurse, integrative und ange- wandte Fragestellungen, konkrete Probleme und lokale Fallstudien (Abb. 4). Diese Themen gruppieren sich dabei um die zwei Schwerpunkte: „globale Agrarwirtschaft" und „Wasser“.

Beim Schwerpunkt „globale Agrarwirtschaft“ spielt Wasser eine untergeordnete Rolle, tritt jedoch bei einem Fallbeispiel zur Integration lokaler in globale Märkte (Anbau der Bio-Mango in Ghana) in Erscheinung. Dabei geht es konkret um die Frage der Bewässerung und der Organisation und Finanzierung dieser (Ouma 2015). Der Übergang zum Schwerpunkt Wasser erfolgt in der Fernerkundung. Mit Mitteln der Fernerkundung lassen sich die Auswirkungen des Wegbleibens bzw. der Zugabe von Wasser im Zusammenhang mit großräumigen Landwirtschafts- und Bewässerungsprojekten und die damit einhergehenden, großräumigen Landschaftsveränderungen effektiv erfassen. Die Desertifikation des Aralsees oder die Bewässerung des Wadi-As-Sirhan Beckens in SaudiArabien dienen dabei als Beispiele (vgl. Abb. 5). In weiteren Lektionen setzen wir uns mit der Wassernutzung in der Schweiz auseinander (hydroklimatische und soziale Aspekte) und mit dem Phänomen Peak Water anhand eines Fallbeispiels aus Peru. Diese Doppellektion Peak Water in Peru werden wir im Folgenden als Beispiel einer pragmatisch-integrativen Lektion detaillierter darlegen.

\section{Doppellektion zu Peak Water in Peru}

Die Doppellektion zu Peak Water in Peru (90 min.) widmet sich den hydroklimatischen Bedingungen in Peru, genauer dem Einzugsgebiet des Rio Santa sowie konkreten Beispielen von Bewässerungsprojekten der Export-Landwirtschaft und damit verbundenen sozioökonomischen und ökologischen Herausforderungen. Die Materialien stammen zum Teil aus laufenden

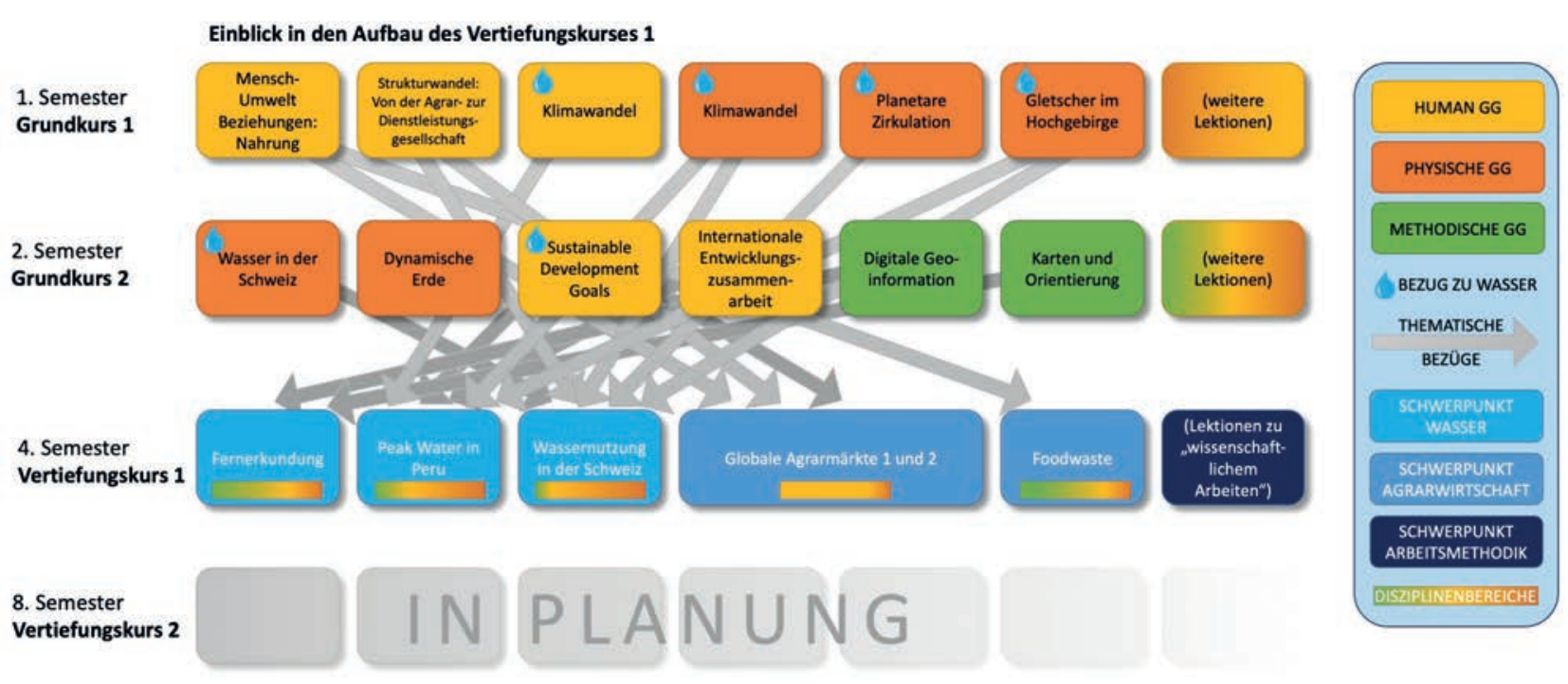

Abb. 4: Querbezüge zwischen den Themen der Grundkurse (oben, vgl. Abb. 2) und den Inhalten des Vertiefungskurses 1 (unten). 

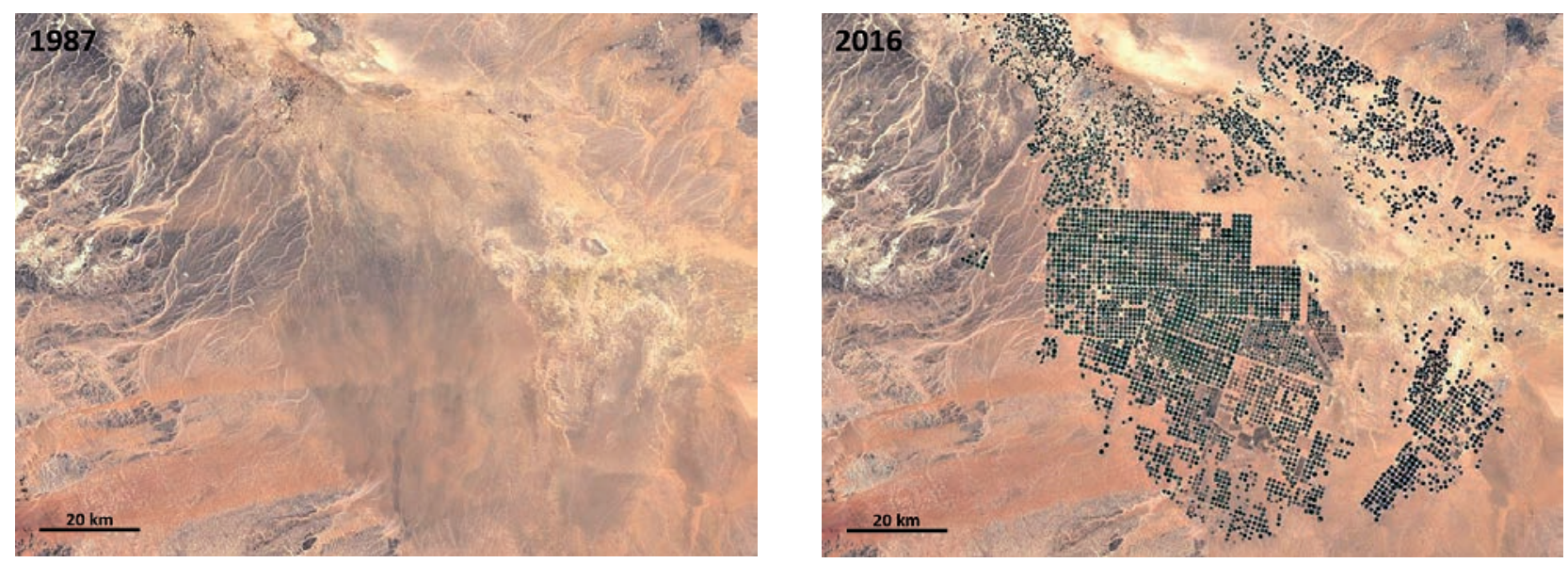

Abb. 5: Satellitenbilder aus den Jahren 1987 und 2016, zeigen den Effekt der Bewässerung und den damit einhergehenden Aufstieg eines Landwirtschaftsimperiums in der Wïste Saudi-Arabiens. (Quelle: eigene Darstellung, basierend auf Bildmaterial von Google Earth, inspiriert von Spiegel online 2012; National Geographic 2018)

inter- und transdisziplinären ${ }^{4}$ Zusammenarbeitsprojekten, die wir in unserer Forschung bearbeiten, und sind somit sehr angewandt.

Die Lernziele dieser Lektion sind:

- Sie können die Veränderungen des Abflusses als Folge des Gletscherrückzuges in einem vergletscherten Einzugsgebiet herleiten und beschreiben, bezogen auf den jährlichen wie auch den saisonalen Abfluss.

- Sie verstehen das Peak Water-Konzept und kennen die Faktoren, die den Zeitpunkt des Peak Water beeinflussen.

- Sie haben eine Vorstellung der Bedeutung verschiedener Landschaftselemente für die andine Quechua-Kultur.

- Sie können Ursachen für die häufigen Konflikte um die Ressource Wasser in Peru nennen und diese an einem Beispiel erläutern.

- Sie kennen das Funktionskonzept des Bewässerungsprojektes Chavimochic an der Peruanischen Küste und können auf verschiedenen Ebenen (regional, national, international) sozioökonomische Vor- und Nachteile des Projektes benennen.

- Sie können Pro- und Kontraargumente für eine Beteiligung von Organisationen der internationalen Entwicklungszusammenarbeit an Projekten wie Chavimochic abwägen.

\footnotetext{
Unter „Interdisziplinarität“ wird der Austausch und die Zusammenarbeit zwischen verschiedenen wissenschaftlichen Disziplinen, Fächern und Fachrichtungen verstanden. „Transdisziplinarität" hingegen beschreibt Unterfangen, die über die Grenzen der rein akademischen Forschung hinausgehen und akademisches mit praktischem Wissen verbindet, um gesellschafts- und/oder politikrelevante Problemstellungen zu bearbeiten. Dies schließt auch die dazu nötigen, besonderen Formen der Kollaboration, Differenzierung und Integration mit ein, ebenso wie die Kommunikation mit der Bevölkerung. (vgl. Bergmann et al. 2005; Backhaus 2008)
}

$\mathrm{Zu}$ Beginn wird an die Themen „Wasser“, „Gletscher", und „Klimatologie“ der Grundkurse angeknüpft und der Beitrag der Gletscherschmelze zum Abfluss aus den tropischen Anden besprochen. Basierend auf dieser Einführung, die gleichzeitig als Auffrischung des bereits behandelten Stoffes dient, erarbeiten sich die Studierenden selbstständig das Konzept des Peak Water, also des Zeitpunkts des maximalen Abflussüberschusses durch abschmelzende Eisreserven (vgl. Baraer et al. 2009; Drenkhan et al. 2018; Huss \& Hock 2018) und damit die zu erwartenden Veränderungen der zukünftigen Wasserverfügbarkeit in vergletscherten Einzugsgebieten. Im Anschluss an diesen physisch-geographischen Einstieg wird auf die Bedeutung der Landschaft in der traditionellen andinen Quechua-Kultur eingegangen und die Perzeption der Landschaftselemente Gletscher, Seen und Flüsse anhand von Bräuchen und Riten besprochen (Abb. 6 links, Mujica 2017) und so die Grundlage für das Verständnis der zahlreichen sozialen Konflikte um das Wasser geschaffen (Bury et al. 2011; Oré \& Muñoz 2018). Diese komplex miteinander verflochtenen Probleme werden am Beispiel des Konfliktes, zwischen einer ausländischen Wasserkraftfirma und einer lokalen Bauerngemeinde, um die Nutzung des Gletschersees Laguna Parón genauer beleuchtet.

Der zweite Teil der Doppellektion widmet sich dem Chavimochic Bewässerungsprojekt an der ariden Pazifikküste, direkt nördlich der Mündung des Santa Flusses. Bei dieser Bewässerungsinfrastruktur wird dem Rio Santa Wasser entnommen, welches der Bewässerung von neu geschaffenen Landwirtschaftsflächen in der Wüste dient, wo internationale AgroKonzerne vor allem Avocados und Spargel, aber auch Zuckerrohr und exotische Früchte anbauen, die in erster Linie für den Export bestimmt sind (Abb. 6 rechts). 
Zuerst wird an die vorangehende Lektion zur Fernerkundung angeknüpft und auf Satellitenbildern die zunehmende Ausdehnung der bewässerten agrarwirtschaftlichen Flächen in dieser Region betrachtet. Im Anschluss wird ein Ausschnitt aus einem Werbefilm gezeigt (Chavimochic 2015), produziert von der Region La Libertad, auf deren Territorium das Gebiet liegt. In diesem Film werden die Vorzüge des Projektes hervorgehoben, wie neu geschaffene Arbeitsplätze und Infrastruktur (Kraftwerke und Kläranlagen). Auch die Bedeutung der exportierten Agrargüter für das Bruttosozialprodukt Perus wird aus wirtschaftlicher Sicht dargestellt. Ergänzt wird dieser Film durch einen Evaluationsbericht einer Deutschen Entwicklungsbank (DEG 2018), die in eine der produzierenden Firmen investiert hat. Dieser Bericht (die Studierenden konzentrieren sich nur auf das Executive Summary), streicht ebenfalls die Nachhaltigkeit sowie den Nutzen für die lokale Bevölkerung hervor und nimmt Bezug auf die nachhaltigen Entwicklungsziele (SDGs), die bereits im Grundkurs behandelt wurden (siehe Kapitel 3.2). Als Gegenperspektive dient ein Filmausschnitt aus einer kritischen Fernsehreportage des Senders arte, der die Nachhaltigkeit des Projektes anzweifelt (arte 2018) sowie zwei Ausschnitte aus einem (von Spektrum.de übersetzten) nature-Artikel von Fraser (2012). Die Texte beleuchten die Schicksale der Saisonarbeiter/innen, die für die Ernte aus den Anden an die Küste kommen und somit dem Wasser folgen.

Zum Abschluss der Doppellektion wird diskutiert, ob sich die Schweiz über das Staatssekretariat für Wirtschaft (SECO) an den Investitionen für einen aktuell zur Diskussion stehenden Ausbau dieses Projektes beteiligen soll oder nicht. Dabei müssen sich die Studierenden für eine Pro- bzw. Kontra-Meinung zu dieser Frage bekennen. Bei dieser Diskussion kommen viele Querbezüge zur Grundkurslektion zur „Internationalen Entwicklungszusammenarbeit" auf. Ganz am Ende wird anhand des Bildes einer Avocado aufgezeigt, wie mit dem Export einer Avocado virtuell große Wassermengen aus einem Wüstengebiet in die wasserreiche Schweiz exportiert werden, was diese tropische Frucht mit der Gletscherschmelze zu tun hat und welche sozialen und wirtschaftlichen Auswirkungen ihr Anbau für die lokale Bevölkerung an der Küste und in den Anden Perus hat.

\subsection{Zwischenfazit Wasser im Vertiefungskurs}

Im Vertiefungskurs werden unter der Thematik „Wasser" angewandte Probleme und Themen integrativ behandelt (siehe Abb. 4). Das Beispiel Peak Water Peru erläuterten wir im obigen Abschnitt deshalb so ausführlich, weil es sehr schön veranschaulicht, wie wir unsere konzeptionellen Gedanken in Bezug auf eine "pragmatisch-integrative Geographie“ konkret in der Hochschullehre umsetzen. Dabei können und wollen wir als Dozierende unseren fachlichen Hintergrund bzw. unsere Herkunft weder verbergen noch verändern: So steigt der physiogeographische Dozent bei der Lektion Peak Water in Peru anhand eines rein glazial-hydrologischen Themas in die Lektion ein. Im Verlauf der Lektion aber werden, im Sinne eines pragmatisch-integrativen Ansatzes, die Querbezüge zu den jeweils anderen Fachbereichen hergestellt. Beim Beispiel Peak Water in Peru geschieht dies in erster Linie aufgrund persönlicher Arbeitserfahrungen in einem interdisziplinären Forschungsprojekt und aus der dabei entstandenen direkten Zusammenarbeit mit der lokalen Bevölkerung einerseits und Erkenntnissen und Einsichten aus Projekten der internationalen Entwicklungszusammenarbeit andererseits. Im Unterricht geht es bei diesen für die Dozierenden
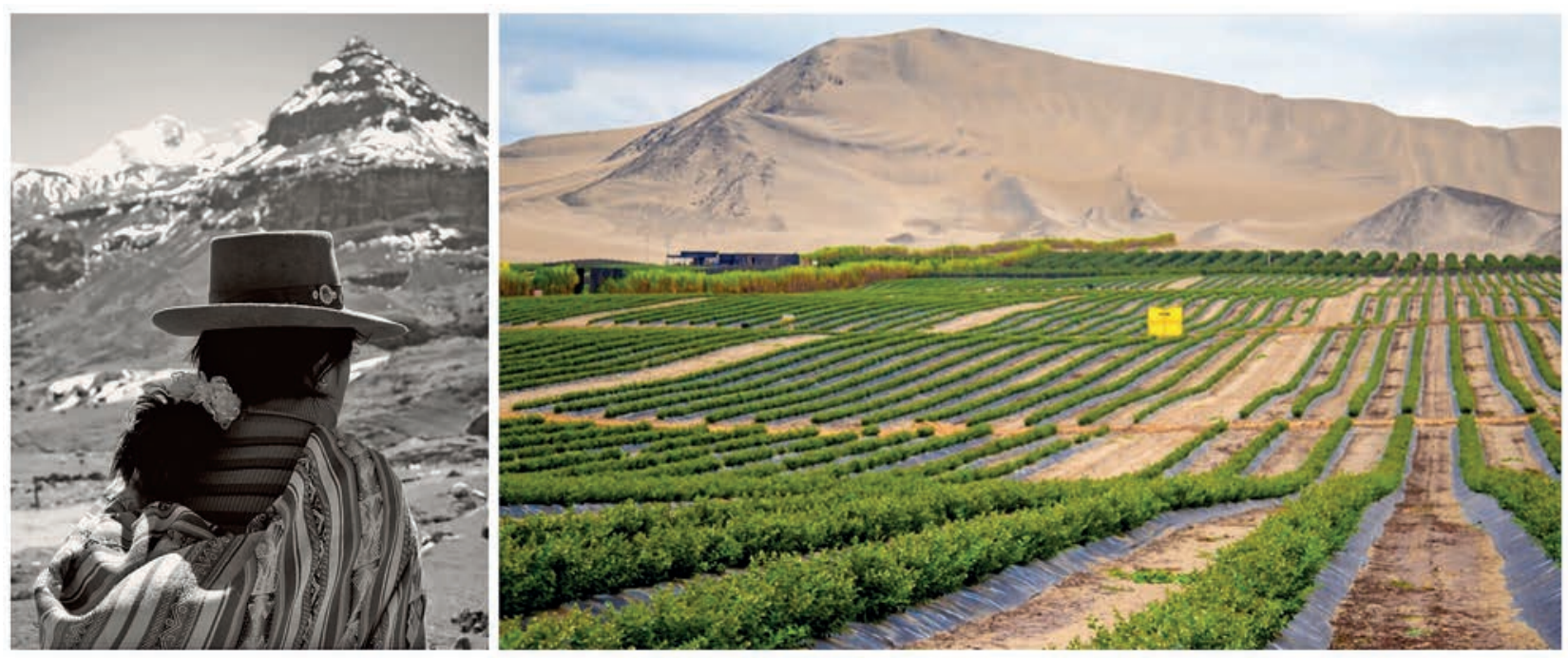

Abb. 6: Links: Eine Frau in der typischen Kleidung der Quechua, die stark vom Gletscherrückgang betroffen sind (Foto: F. Drenkhan). Rechts: Extensive Bewässerung mit Wasser aus den Anden ermöglicht Großlandwirtschaft im ariden Küstenstreifen am Pazifik (Foto: La Industria). 
„fachfremden" Aspekten weniger um den theoretischen Unterbau (dieser ist bereits in den Grundkursen gelegt worden), sondern hauptsächlich um eine Perspektivenerweiterung, die einer integrativen Betrachtung der Problematik dienen soll. Die Studierenden können so die Vielschichtigkeit des Themas „Wasserverfügbarkeit und -nutzung" am konkreten Fallbeispiel erfahren und werden sensibilisiert dafür, dass es verschiedene Zugänge braucht, um ein komplexes Problem zu verstehen. Dies bildet sich auch in den Lernzielen der Lektion ab.

Dass wir Dozierenden dabei die Rolle der Humangeographinnen bzw. der Physiogeographen nicht völlig ablegen können, sehen wir nicht als Nachteil. Es geht uns vor allem darum, die Vorteile und Notwendigkeit einer interdisziplinären Herangehensweise an die behandelten, komplexen Real World-Probleme aufzuzeigen, sowohl in der ganzheitlichen Erfassung des Problems, wie auch bei der Erarbeitung möglicher Lösungsansätze. Dabei versuchen wir in erster Linie (nebst der Notwendigkeit eines gewissen allgemeingeographischen Grundwissens) eine Offenheit gegenüber Themen und Ansätzen anderer Fachbereiche vorzuleben, ohne aber unsere fachlichen Neigungen als spezialisierte Forschende zu verschleiern.

\section{$4 \quad$ Diskussion und Ausblick}

In der abschließenden Diskussion möchten wir noch einmal die wichtigsten Punkte zusammenfassen und einen Ausblick auf die weitere Lehrplanung geben.

Wenn wir einen gemeinsamen „Typus“ für uns finden und diesen charakterisieren müssten, dann in etwa so: Pragmatisch, Differenzen in der Forschungsausrichtung anerkennend, aber grundsätzlich interessiert an einem offen geführten Dialog. In den gemeinsam erarbeiteten Lehrgefäßen für angehende Sekundarschullehrpersonen ist es so, dass wir neben Themen, die wir integrativ bearbeiten, auch Themen aus physisch-geographischer sowie humangeographischer Perspektive unterrichten. Dennoch ist es uns ein Anliegen, dass wir durch die Kooperation voneinander lernen können und ernsthaft versuchen den Lehrpersonen, die später in der Schule für die Einheit des Faches mit ihrer eigenen Person einstehen, eine solche Vielseitigkeit vorzuleben. Eine „pragmatisch-integrative Geographie“ ist für uns, so wie wir sie heute in unseren Modulen leben, eine „offene Beziehung“ zwischen unterschiedlichen Partnerinnen und Partnern, bei der wir bewusst auch Themen in den Unterricht integrieren, bei denen physisch- und humangeographische Fragestellungen einander befruchten können oder gar so ineinandergreifen, dass diese nicht (mehr) als getrennte Perspektiven wahrgenommen werden.
Vor allem bei den meist angewandten und praxisnahen Themen des Vertiefungskurses bedarf es unserer Ansicht nach einer „integrativen Perspektive“, um die Probleme überhaupt in ihrer ganzen Komplexität erfassen zu können. Dies korrespondiert auch mit dem Lehrplan 21 (vgl. EDK o. J.), der u. a. festlegt, welche Themen und Kompetenzen in den jeweiligen Fachbereichen an den Deutschschweizer Sekundarschulen unterrichtet werden. Die Geographie ist hier dem Fachbereich „Räume, Zeiten, Gesellschaften“ (Sekundarstufe I) zugeordnet.

Unsere pragmatisch-integrativen Teile des Unterrichts haben sicher dadurch gewonnen, dass wir uns nicht nur im Rahmen von regelmäßigen „Lehrreflexionen" oft ausgetauscht haben. So führten einerseits Nachfragen und Anmerkungen der Humangeographinnen oder der Physiogeographen teilweise zu kleinen Anpassungen des geplanten Inhaltes. Die Lektion „Fernerkundung \& Wasser" begann etwa mit einem Bild, das exakt die Region zeigte, die eine Woche zuvor als Beispiel diente, um die Bewässerungsproblematik der Bio-Mangos in Ghana zu besprechen. Anderseits waren unsere gemeinsame Lehrreflexion und Zusammenarbeit auch Basis für Grundsätzlicheres. So zeigte sich in einer Sitzung zu unserer gemeinsamen Curriculumsentwicklung, dass wir erstens der Meinung sind, dass bestimmte fachliche Grundlagen in der Humangeographie und physischen Geographie erst einmal separat erlernt und eingeübt werden müssen, bevor an geeigneten Themen (z. B. im Grundkurs 1 am Thema „Klimawandel“, oder im Vertiefungskurs 1 am Thema "Wasser") eine verknüpfende Perspektive sowie verschiedene Methoden der Geographie auf einer vertiefenden Ebene erörtert werden können. Selbstkritisch sollten wir uns hier jedoch fragen, ob es tatsächlich die Themen per se sind, die verlangen, dass sie erst einmal separat erlernt werden müssen, oder aber, ob nicht eher Ängste und ein gewisses Widerstreben unsererseits dafür ausschlaggebend sind, dass wir diese Themen so betrachten; Ängste etwa, dass Themen und Aspekte, die uns am Herzen liegen evtl. etwas weniger Raum bekommen würden, wenn wir sie verstärkt aus einer integrativen Perspektive behandeln würden. Oder ein Widerstreben „perfekt" vorbereitete Lektionen ganz neu aus einer integrativen Perspektive zu denken und entsprechend überarbeiten zu müssen. Denn, dass wir feministische Geographie und Glaziologie verbinden könnten, blieb bisher immer nur ein Spruch, obwohl es durchaus Material und Literatur zu einer solchen Verbindung gibt, z. B. Carey et al. (2016). Zweitens entwickelte sich in der Curriculumsplanung weiter die Idee, dass wir in den beiden Vertiefungskursen Themen und Fragestellungen ins Zentrum rücken möchten, die sich 
gut für eine integrative Perspektive eignen - wie das Thema Wasser im Vertiefungskurs 1 oder das Thema Rohstoffe, das für den Vertiefungskurs 2 geplant ist. Solche Entscheide, sei dies nun auf Curriculumsebene oder eher im Kleinen, gemeinsam pragmatisch aber zugleich rücksichtsvoll zu fällen und dann auch entsprechend umzusetzen, sehen wir als bedeutende Voraussetzung für einen pragmatisch-integrativen Unterricht, den alle Beteiligten motiviert mittragen.

Abschließend möchten wir gerne noch auf zwei Aspekte eingehen, die wir selbstkritisch als Herausforderungen und als „Baustellen“ für die weitere Planung und Durchführung unserer Lehrveranstaltungen (v.a. für den Vertiefungskurs 2) sehen.

Erstens, die Doppellektion Peak Water in Peru ist von der thematischen Anlage her bislang wahrscheinlich die ,integrativste“ unserer Lektionen. Interessanterweise wurde sie von einem Physiogeographen erarbeitet und gehalten, der in einem interdisziplinären Forschungsnetzwerk genau zu diesem Thema arbeitet und daher sehr gerne die Einsichten einer integrativen Geographie in die Lehre einbringt. Inhaltlich und konzeptionell entspricht diese Lektion vollauf dem Konzept der „pragmatisch-integrativen Geographie“. Allerdings sehen wir noch Potenzial die humangeographischen Inhalte in diesem Zusammenhang weiter zu vertiefen und im Team auszuarbeiten. Hier sind also die Humangeographinnen am Zug, während in anderen Themenbereichen (z. B. betreffend virtuellem Wasser oder beim Thema food waste) der physiogeographische Anteil noch weiter ausgebaut werden kann.

Zweitens beschäftigte uns immer wieder die Frage des zeitlichen Aufwandes und damit verbunden, ob wir mit der aktuell pragmatisch-ressourcenorientierten Lehraufteilung - nur ein/e Dozent/in pro Lektion - eine Dualität von Humangeographie und physischer Geographie sehr stark verkörpern und dadurch auch permanent reproduzieren. Wie könnte es gelingen, diese Dualität aufzubrechen ohne, dass wir zu zweit im Unterrichtsraum stehen müssen und ohne die Inhalte in fachlicher Hinsicht zu schwächen? Bisher besprechen wir die geplanten Lehrinhalte und didaktischen Methoden teilweise miteinander, führen punktuell kollegiale Hospitationen mit Feedbacks durch, tauschen Fachwissen, Lehr- und Lernmaterialien aus und unterstützen uns gegenseitig in allen Belangen der Lehre (inkl. Prüfungen). Dies brauchte Zeit. Die Lehre im Vertiefungskurs 1 zeigte uns bei der erstmaligen Durchführung (im FS2019) auch, dass wir oft im Gespräch Ideen entwickelten, wie wir den pragmatisch-integrativen Ansatz verstärkt in der Lehre umsetzen könnten, ohne, dass wir diese Ideen dann aber tatsächlich umgesetzt haben. Dies hat ebenfalls ganz entscheidend mit der Ressource Zeit zu tun, die schlussendlich auch Grenzen dieses An- satzes in der Praxis aufzeigt. Ein pragmatischer sowie selbstverantwortlicher Umgang mit unseren zeitlichen Ressourcen ist uns wichtig, um neben der Lehre auch noch Zeit für die eigene Forschungsarbeit zu haben. Die Arbeit an diesem Artikel hat uns diesbezüglich erste Antworten gegeben. Der Zusatz „pragmatisch“ in „pragmatisch-integrativer Geographie“ motiviert uns, andere Wege der Vor- und Nachbereitung bzw. Gestaltung unserer Lehre zu testen: Konkrete Ideen für eine zukünftige, zeiteffiziente (und damit eben auch pragmatische) Umsetzung von ,integrativer Geographie" sehen wir u. a. in der Einbindung von Kurzstatements, Expertisen und Videosequenzen von Kolleginnen und Kollegen aus anderen Fachgebieten. Auch möchten wir den Studierenden an geeigneten Stellen gerne transparenter aufzeigen, welche Diskussionen und Überlegungen auf der „Hinterbühne“ dazu führten, dass ein bestimmtes Lektionsthema inhaltlich und didaktisch in der vorliegenden Form aufbereitet wurde.

Mit unserer Hochschullehre für angehende Sekundarschullehrpersonen, die neben anderen Fachbereichen auch Geographie unterrichten werden, bieten wir ein kompaktes fachwissenschaftliches Kurzstudium in Geographie an, das die Studierenden dazu befähigen und ermuntern soll, Jugendliche später in der Schule für geographische Phänomene und Themen zu begeistern. Für diese zukünftigen Lehrpersonen ist eine Unterscheidung in Physio- oder Humangeographie nicht wirklich relevant. Daher streben wir in der Lehre eine pragmatisch-integrative Geographie an, ohne dabei unsere fachlichen Wurzeln als Human- bzw. Physiogeographinnen und -geographen künstlich zu kaschieren. Die fachliche und geographiedidaktische Reflexion der eigenen Lehre im Team hat den Diskussionen neue Impulse gegeben, weil wir gemeinsam das Konzept einer „pragmatisch-integrativen Geographie“ entwickelten und direkt in der Lehrpraxis testeten und weiter vorantreiben. Die Verknüpfung von fachlichen Hintergründen, gemeinsamer Lehre und didaktischer Reflexion resultierte in einem kooperativen Schreibprozess (vgl. Bauer \& Keller 2018), der in dieser Form ein Novum zumindest an unserem Institut darstellt und zu einer Intensivierung des Dialogs unter Geographinnen und Geographen sowie Geographiedidaktikerinnen und -didaktikern aufrufen möchte.

\section{Literatur}

arte (2018): Klimawandel: Wenn das Gletschereis schmilzt. https://www.arte.tv/de/videos/081896000-A/klimawandel-wenn-das-gletschereis-schmilzt/ (20.08.2019) 
Backhaus, N. (2008): Macht und Kraft der Bilder: ein (preisgekröntes) Beispiel transdisziplinärer Forschung. In: Darbellay, F. \& T. Paulsen (Hrsg.): Le défi de l'inter- et transdisciplinarité $=$ Herausforderung Inter- und Transdisziplinarität. S. 239-260.

Baraer, M., J. M. McKenzie, B. G. Mark et al. (2009): Characterizing contributions of glacier melt and groundwater during the dry season in a poorly gauged catchment of the Cordillera Blanca (Peru). In: Advances in Geosciences 22. S. 41-49.

Bauer, I. (2015): Akteur-Netzwerk-Theorie im Geographieunterricht? Das Beispiel „Wasser“. In: Gryl, I., A. Schlottmann \& D. Kanwischer (Hrsg.): Mensch:Umwelt:System - Theoretische Grundlagen und praktische Beispiele für den Geographieunterricht. S. 251-266.

Bauer, I. \& I. Gryl (2018): Quo vadis Geographiedidaktik (II): Was die Fishbowl-Diskussion auf dem HGDSymposium in Jena (2017) an Perspektiven und Grenzen aufzeigte. In: GW-Unterricht. S. 20-33.

Bauer, I. \& M. Keller (2018): „Opening up spaces for thought": Konstruktiver Dialog zwischen Geographie und Geographiedidaktik am Beispiel Kinder- und Jugendgeographien. In: OpenSpaces. Zeitschrift für Didaktiken der Geographie. S. 7-24.

Bergmann, M., B. Brohmann, E. Hofmann et al. (2005): Quality Criteria of Transdisciplinary Research. A Guide for the Formative Evaluation of Research Projects. With a Foreword by Thomas Jahn. ISOE-Studientexte 13 . Frankfurt am Main: ISOE - Institut für sozial-ökologische Forschung.

Bury, J. T., B. G. Mark, J. M. McKenzie et al. (2011): Glacier recession and human vulnerability in the Yanamarey watershed of the Cordillera Blanca, Peru. In: Climatic Change 105. S. 179-206.

Carey, M., M. Jackson, A. Antonello et al. (2016): Glaciers, gender, and science: A feminist glaciology framework for global environmental change research. In: Progress in Human Geography 40(6). S. 770-793.

Castree, N. (2015): Changing the Anthropo(s)cene: Geographers, global environmental change and the politics of knowledge. In: Dialogues in Human Geography 3(3). S. 310-316.

Chavimochic (2015): Video Institucional Proyecto Especial. https://www.youtube.com/watch?v=6vZKCR4oy_0 (20.08.2019)

Crutzen, P. J. (2002): Geology of mankind. In: Nature 415. S. 23.

DEG (2018): Evaluierungsergebnisse der DEG - Beitrag von Virú zur nachhaltigen Entwicklung von Peru. https://www.deginvest.de/DEG-Dokumente/Über-uns/ Was-wir-bewirken/DE_Case-Study_Viru_2018_final. $\operatorname{pdf}(20.08 .2019)$

Dickel, M. \& F. Pettig (2015): Landschaft als kulturgeschichtliches Phänomen. Über die Didaktisierung einer Seh- und Denkfigur. In: Gryl, I., A. Schlottmann \& D. Kanwischer (Hrsg.): Mensch:Umwelt:System - Theoreti- sche Grundlagen und praktische Beispiele für den Geographieunterricht. S. 235-250.

Drenkhan, F., L. Guardamino, C. Huggel \& H. Frey (2018): Current and future glacier and lake assessment in the deglaciating Vilcanota-Urubamba basin, Peruvian Andes. In: Global and Planetary Change 169. S. 105-118. DOI: 10.1016/j.gloplacha.2018.07.005.

EDA (2017): Ziel 6: Verfügbarkeit und nachhaltige Bewirtschaftung von Wasser und Sanitärversorgung für alle gewährleisten. https://www.eda.admin.ch/agenda2030/ de/home/agenda-2030/die-17-ziele-fuer-eine-nachhaltige-entwicklung/ziel-6-verfuegbarkeit-und-nachhaltigebewirtschaftung-von-wasser.html Zugriff: (20.8.2019)

EDK (o. J.): Lehrplan 21. https//lehrplan21.ch (20.8.2019)

Egli, H.-R., M. Hasler \& M. Probst (Hrsg.) (2016): Geographie. Wissen und Verstehen. hep, Bern.

Fraser, B. (2012): Melting in the Andes: Goodbye glaciers. In: Nature 491. S. 180-182.

Gebhardt, H. et al. (Hrsg.) (2012): Geographie. Physische Geographie und Humangeographie. Spektrum, Heidelberg. S. 1086-7.

Geist, H. J. (2018): Integrative Geographie neu denken - z. B. anthropozänisch. In: geographica helvetica 73 . S. 187-191.

Gryl, I., A. Schlottmann \& D. Kanwischer (Hrsg.) (2015): Mensch:Umwelt:System - Theoretische Grundlagen und praktische Beispiele für den Geographieunterricht. Lit, Berlin.

Heinritz, G. (Hrsg.) (2003): Integrative Ansätze in der Geographie - Vorbild oder Trugbild? Münchner Symposium zur Zukunft der Geographie, 28.4.2003. Eine Dokumentation. Münchner Geographische Hefte 85. Passau.

Helvetas (2019): https://www.helvetas.org/de/schweiz/werwir-sind/vision-engagement (20.8.2019)

Huss, M. \& R. Hock (2018): Global-scale hydrological response to future glacier mass loss. In: Nature Climate Change 8. S. 135-140.

Johnson, E. \& H. Morehouse (2014): After the Anthropocene: Politics and geographic inquiry for a new epoch. In: Progress in Human Geography, 38(3). S. 439-456.

Kanwischer, D. (2006): Der Doppelcharakter der Geographie und andere Katastrophen nebst einigen Bemerkungen zur fachdidaktischen Umsetzung. In: Horst, U., D. Kanwischer \& D. Stratenwerth (Hrsg.): Die Kunst sich einzumischen. Vom vielfältigen und kreativen Wirken des Geographen Tilman Rhode-Jüchtern. S. 127-142.

Kersting, D. (2015): Ein kritischer Überblick systemtheoretisch geprägter Ansätze in der Geographie. In: Gryl, I., A. Schlottmann \& D. Kanwischer (Hrsg.): Mensch:Umwelt:System - Theoretische Grundlagen und praktische Beispiele für den Geographieunterricht. S. 43-60.

Knitter, D. et al. (2019): Geography and the Anthropocene: Critical approaches needed. In: Progress in Physical Geography 43. S. 451-461. DOI: 10.1177/0309133319829395. 
Lave, R., M. W. Wilson, C. Biermann et al. (2014): Intervention: Critical physical geography. In: The Canadian Geographer 58. S. 1-10.

Lave, R., C. Biermann \& S. N. Lane (Hrsg.) (2018): The Palgrave Handbook of Critical Physical Geography. Palgrave Macmillan, Cham.

Mehren, R., A. Rempfler, E. M. Ulrich-Riedhammer et al. (2015): Validierung eines Kompetenzmodells zur Geographischen Systemkompetenz. In: Gryl, I., A. Schlottmann \& D. Kanwischer (Hrsg.): Mensch:Umwelt:System - Theoretische Grundlagen und praktische Beispiele für den Geographieunterricht. S. 61-82.

Müller-Mahn, D. \& U. Wardenga (2005): Möglichkeiten und Grenzen integrativer Forschungsansätze in Physischer Geographie und Humangeographie. Leibniz-Institut für Länderkunde, Leipzig.

Mujica, L. F. (2017): Pachamama kawsan: hacia una ecologia andina. PUCP - UNAJMA, Lima.

National Geographic (2018): Wasser, Nahrung, Energie Die Wüsten werden grün. https://www.nationalgeographic.de/umwelt/2018/04/wasser-nahrung-energie-diewuesten-werden-gruen (11.03.2019)

Neuer, B. \& A. Schlottmann (2014): Zur Bedeutung der Fachdidaktik in der deutschsprachigen Geographie. In: Editorial Rundbrief Geographie 250. S. 1-3.

Oré, M. T. \& I. Muñoz (2018): Aguas en disputa. Ica y Huancavelica, entre el entrampamiento y el diálogo. PUCP, Lima.

Ouma, S. (2015): Assembling Export Markets: The Making and Unmaking of Global Food Connections in West Africa. Wiley, o. O.

PHZH(o.J.):FächerwahldesMasterVollzeit-undTeilzeitstudiums Sekundarstufe I. https://phzh.ch/de/Ausbildung/ Studiengaenge/Sekundarstufe1/Integrierter-Masterstudiengang-Sekundarstufe1/Faecherwahl/ (05.12.2019)

Rockström J., W. Steffen, K. Noone et al. (2009): Planetary boundaries: exploring the safe operating space for humanity. In: Ecology and Society 14. S. 1-24.

Schlottmann, A., B. Braun, A. Bräuning et al. (2018): Editorial im Rundbrief Geographie 274. S. 1-4.

Schlottmann, A. (2015): (Wie) ist „Systemkompetenz“ möglich? Humangeographische, erkenntnistheoretische und pragmatische Perspektiven für eine integrative geographische Bildung. In: Gryl, I., A. Schlottmann \& D. Kanwischer (Hrsg.): Mensch:Umwelt:System - Theoretische Grundlagen und praktische Beispiele für den Geographieunterricht. S. 99-128.

Schneider, A. \& J. L. Wilhelm (2015): Systeme konkret. Spielend systemisch denken. In: Gryl, I., A. Schlottmann \& D. Kanwischer (Hrsg.): Mensch:Umwelt:System -
Theoretische Grundlagen und praktische Beispiele für den Geographieunterricht. S. 131-145.

Spiegel online (2012): Satellitenbild der Woche: Nasser Wüstensand - Fotostrecke http://www.spiegel.de/fotostrecke/ satellitenfotos-zeigen-wie-saudi-arabien-die-wuestebewirtschaftet-fotostrecke-80690.html

(11.03.2019)

Steinbrink, M. \& P. Aufenvenne (2016): Integrative Geographiedidaktik? Versuch einer Positionsbestimmung der Fachdidaktik innerhalb der deutschsprachigen Geographie. In: GW-Unterricht 142/143. S. 5-17.

Steiner, C. (2014): Von Interaktion zu Transaktion - Konsequenzen eines pragmatischen Mensch-Umwelt-Verständnisses für eine Geographie der Mitwelt. In: Geographica Helvetica 69(1). S. 171-181.

UN (o. J.): https://sustainabledevelopment.un.org/sdg6 (11.03.2019)

Wardenga, U. (2005): Wozu Erinnerung? Über die Rolle von Fachgeschichtsbildern in der Debatte um integrative Forschungsansätze in der Geographie. In: Müller-Mahn, D. \& U. Wardenga: Möglichkeiten und Grenzen integrativer Forschungsansätze in Physischer Geographie und Humangeographie. Leibniz-Institut für Länderkunde, Leipzig. S. 7-36.

Wardenga, U. \& P. Weichhart (2006): Auf dem Weg zur „Dritten Säule“. Sozialökologische Interaktionsmodelle und Systemtheorien - Ansätze einer theoretischen Begründung integrativer Projekte in der Geographie? In: Mitteilungen der Österreichischen Geographischen Gesellschaft 148. S. 9-31.

Wardenga, U. \& P. Weichhart (2012): Schnittstellenforschung. In: Gebhardt, H. et al. (Hrsg.): Geographie. Physische Geographie und Humangeographie. Spektrum, Heidelberg. S. 1086-7.

Weichhart, P. (2005): Auf der Suche nach der «dritten Säule». Gibt es Wege von der Rhetorik zur Pragmatik? In: Müller-Mahn, D. \& U. Wardenga (Hrsg.): Möglichkeiten und Grenzen integrativer Forschungsansätze in Physischer Geographie und Humangeographie. (=forum ifl 2). Leibniz-Institut für Länderkunde, Leipzig. S. 109-136.

Weichhart, P. (2003): Physische Geographie und Humangeographie - eine schwierige Beziehung: Skeptische Anmerkungen zu einer Grundfrage der Geographie und zum Münchner Projekt einer „Integrativen Umweltwissenschaft “. Integrative Ansätze in der Geographie - Vorbild oder Trugbild. S. 17-34.

Wesselink, A., M. Kooy \& J. Warner (2017): Socio-hydrology and hydrosocial analysis: toward dialogues across disciplines. In: WIREs Water 4(1196). S. 1-14. doi: 10.1002/wat2.1196. 